\title{
POSITIVE PARTIAL REALIZATION PROBLEM FOR LINEAR DISCRETE-TIME SYSTEMS
}

\author{
TADEUSZ KACZOREK \\ Faculty of Electrical Engineering \\ Białystok Technical University \\ Wiejska 45D, 15-351 Białystok \\ e-mail:kaczorek@isep.pw.edu.pl
}

\begin{abstract}
A partial realization problem for positive linear discrete-time systems is addressed. Sufficient conditions for the existence of its solution are established. A procedure for the computation of a positive partial realization for a given finite sequence of the values of the impulse response is proposed. The procedure is illustrated by four numerical examples.
\end{abstract}

Keywords: positive systems, partial realization, existence, computation, linear systems, discrete-time systems

\section{Introduction}

In positive systems, the inputs, state variables and outputs take only non-negative values. Examples of positive systems are industrial processes involving chemical reactors, heat exchangers and distillation columns, storage systems, compartmental systems, or water and atmospheric pollution models. A variety of models having positive linear system behaviour can be found in engineering, management science, economics, social sciences, biology and medicine, etc.

Positive linear systems are defined on cones and not on linear spaces. Therefore, the theory of positive systems is more complicated and less advanced. Overviews of the state of the art in positive systems theory are given in the monographs (Farina and Rinaldi, 2000; Kaczorek, 2002). The realization problem for positive linear systems was considered in many papers and books (Benvenuti and Farina, 2004; Farina and Rinaldi, 2000; Kaczorek, 1992; Kaczorek, 2002; Kaczorek, 2006a; Kaczorek, 2006b; Kaczorek, 2004; Kaczorek, 2005; Kaczorek, 2006c; Kaczorek, 2006d; Kaczorek, 2006e; Kaczorek and Busłowicz, 2004).

The problem of constructing linear state variable models from given impulse responses (or Markov parameters) was considered in (Ho and Kalman, 1966; Kaczorek, 1992). The partial realization problem consists in constructing state variables models from given finite sequences of impulse responses.
In this paper the partial realization problem will be addressed for positive linear discrete-time systems. Sufficient conditions for the existence of its solution will be established and a procedure for the computation of its positive realization for a given finite sequence of the values of the impulse response will be proposed. To the best of the author's knowledge, the positive partial realization problem for discrete-time linear system has not been considered yet.

\section{Preliminaries and Problem Formulation}

Let $\mathbb{R}^{n \times m}$ be the set of $n \times m$ real matrices and $\mathbb{R}^{n}:=$ $\mathbb{R}^{n \times 1}$. The set of $n \times m$ real matrices with non-negative entries will be denoted by $\mathbb{R}_{+}^{n \times m}$ and the set of nonnegative integers by $\mathbb{Z}_{+}$.

Consider the linear discrete-time system

$$
\begin{aligned}
x_{i+1} & =A x_{i}+B u_{i}, \quad i \in \mathbb{Z}_{+}, \\
y_{i} & =C x_{i}+D u_{i},
\end{aligned}
$$

where $x_{i} \in \mathbb{R}^{n}, u_{i} \in \mathbb{R}^{m}, y_{i} \in \mathbb{R}^{p}$ are the state, input and output vectors, and $A \in \mathbb{R}^{n \times n}, B \in \mathbb{R}^{n \times m}, C \in \mathbb{R}^{p \times n}$ and $D \in \mathbb{R}^{p \times m}$.

The system (1) is called (internally) positive if and only if $x_{i} \in \mathbb{R}_{+}^{n}$ and $y_{i} \in \mathbb{R}_{+}^{p}, i \in \mathbb{Z}_{+}$for every $x_{0} \in \mathbb{R}_{+}^{n}$ and any input sequence $u_{i} \in \mathbb{R}_{+}^{m}, i \in \mathbb{Z}_{+}$. The system (1) is (internally) positive if and only if (Kaczorek, 2002; Fa- 
rina and Rinaldi, 2000):

$$
A \in \mathbb{R}_{+}^{n \times n}, \quad B \in \mathbb{R}_{+}^{n \times m}, C \in \mathbb{R}_{+}^{p \times n}, \quad D \in \mathbb{R}_{+}^{p \times m} .
$$

The transfer matrix of (1) is given by

$$
T(z)=C[I z-A]^{-1} B+D=C[I-A d]^{-1} d B+D,
$$

where $d=z^{-1}$ is the delay operator.

Let

$$
G(d)=\sum_{i=0}^{\infty} G_{i} d^{i}
$$

be the impulse response matrix of the system (1). From the well-known equality

$$
G_{i}= \begin{cases}D & \text { for } i=0, \\ C A^{i-1} B & \text { for } i=1,2, \ldots\end{cases}
$$

and (2), it follows that for the positive system (1) we have that $G_{i} \in \mathbb{R}_{+}^{p \times m}$ for $i \in \mathbb{Z}_{+}$

The partial positive realization problem can be formulated as follows: Given a finite sequence of nonnegative matrices

$$
\hat{G}_{i} \in \mathbb{R}_{+}^{p \times m} \quad \text { for } \quad i=0,1, \ldots, N,
$$

find the matrices (2) of the positive system (1) such that

$$
\hat{G}_{i}=G_{i} \quad \text { for } \quad i=0,1, \ldots, N,
$$

with $G_{i}$ defined by (4).

In this paper sufficient conditions for the existence of a solution to the above problem will be established and a procedure for the computation of the matrices (2) for a given finite sequence $G_{i} \in \mathbb{R}_{+}^{p \times m}, i=0,1, \ldots, N$ will be proposed.

\section{Problem Solution}

First, the idea of the proposed method will be outlined for a single-input single-output (SISO $m=p=1$ ) system. In this case the transfer function of (1) can be written down in the form

$$
T(d)=\frac{b(d)}{a(d)}=\frac{\sum_{i=0}^{n} b_{i} d^{i}}{1-\sum_{i=1}^{n} a_{i} d^{i}},
$$

and its impulse response is

$$
g(d)=\sum_{i=0}^{\infty} g_{i} d^{i}, \quad g_{i} \in \mathbb{R}_{+}, \quad i \in \mathbb{Z}_{+} .
$$

The partial positive realization problem can be decomposed into the following two subproblems:
Subproblem 1. Given a finite sequence

$$
\hat{g}_{i} \geq 0 \text { for } i=0,1, \ldots, N
$$

find $n \leq N$ and the transfer function (8) such that

$$
\hat{g}_{i}=g_{i} \quad \text { for } \quad i=0,1, \ldots, N
$$

where $g_{i}$ is defined by (9).

Subproblem 2. Given the transfer function (8), find its positive realization (2).

3.1. Solution of Subproblem 1. Taking into account that

$$
T(d)=\sum_{i=0}^{\infty} g_{i} d^{i}
$$

and using (8), we can write

$$
a(d) g(d)=b(d)
$$

and

$$
a(d)[\hat{g}(d)+\bar{g}(d)]=b(d)
$$

where the polynomial

$$
\hat{g}(d)=\sum_{i=0}^{N} \hat{g}_{i} d^{i}
$$

is known and the sum

$$
\bar{g}(d)=\sum_{i=N+1}^{\infty} \bar{g}_{i} d^{i}
$$

is unknown.

Equation (14) can be rewritten in the form

$$
a(d) \hat{g}(d)=b(d)+\tilde{g}(d),
$$

where

$$
\tilde{g}(d)=-a(d) \bar{g}(d)=\sum_{i=N+1}^{\infty} \tilde{g}_{i} d^{i} .
$$

The subproblem has thus been reduced to the following one: Given the polynomial (15), find the polynomials $a(d)$ and $b(d)$ of (8) such that (17) holds for some $\tilde{g}(d)$. 
Using (8) and (15), we obtain

$$
\begin{aligned}
a(d) \hat{g}(d)= & \left(1-\sum_{i=1}^{n} a_{i} d^{i}\right)\left(\sum_{j=0}^{N} \hat{g}_{j} d^{j}\right) \\
= & \sum_{j=0}^{N} \hat{g}_{j} d^{j}-\sum_{i=1}^{n} \sum_{k=i}^{i+N} a_{i} \hat{g}_{k-i} d^{k} \\
= & \sum_{k=0}^{N}\left(\hat{g}_{k}-\sum_{i=1}^{n} a_{i} \hat{g}_{k-i}\right) d^{k} \\
& +\sum_{k=N+1}^{N+n}\left(\sum_{i=k-N}^{n} a_{i} \hat{g}_{k-i}\right) d^{k} \\
= & \sum_{k=0}^{N} q_{k} d^{k}+\sum_{k=N+1}^{N+n}\left(\sum_{i=k-N}^{n} a_{i} \hat{g}_{k-i}\right) d^{k},
\end{aligned}
$$

where

$$
q_{k}=\hat{g}_{k}-\sum_{i=1}^{n} \hat{g}_{k-i} a_{i}
$$

Let $q_{k}=0$ for $k=n+1, \ldots, N$. Then from (20) we have

$$
\hat{G}_{n N} a=\hat{g}_{n N}
$$

where

$$
\begin{gathered}
\hat{G}_{n N}=\left[\begin{array}{cccc}
\hat{g}_{n} & \hat{g}_{n-1} & \ldots & \hat{g}_{1} \\
\hat{g}_{n+1} & \hat{g}_{n} & \ldots & \hat{g}_{2} \\
\ldots & \ldots & \ldots & \ldots \\
\hat{g}_{N-1} & \hat{g}_{N-2} & \ldots & \hat{g}_{N-n}
\end{array}\right], \\
a=\left[\begin{array}{c}
a_{1} \\
a_{2} \\
\vdots \\
a_{n}
\end{array}\right], \quad \hat{g}_{n N}=\left[\begin{array}{c}
\hat{g}_{n+1} \\
\hat{g}_{n+2} \\
\vdots \\
\hat{g}_{N}
\end{array}\right] .
\end{gathered}
$$

We assume that

$$
\operatorname{rank} \hat{G}_{n N}=\operatorname{rank}\left[\hat{G}_{n N}, \hat{g}_{n N}\right],
$$

and Eqn. (21) has a non-negative solution, i.e., $a \in \mathbb{R}_{+}^{n}$. In (Kaczorek, 2006e), a method was proposed for the computation of a positive solution to (21). Note that (19) has the form (17) if and only if $q_{k}=0$ for $k=n+1, \ldots, N$ or, equivalently, the coefficients $a_{1}, a_{2}, \ldots, a_{n}$ of the polynomial $a(d)$ are a solution to (21). Knowing the coefficients $a_{i}, i=0,1, \ldots, n$, we can compute the coefficients $b_{j}, j=0,1, \ldots, n$ of the polynomial $b(d)$ using the formula

$$
b_{k}=\hat{g}_{k}-\sum_{i=1}^{n} \hat{g}_{k-i} a_{i} \quad \text { for } \quad k=0,1, \ldots, n .
$$

It follows from the comparison of the right-hand sides of (17) and (19) for $q_{k}=0, k=n+1, \ldots, N$.
In summary, we have the following result:

Theorem 1. Let $n \leq N$. Given a finite sequence (10), there exists a transfer function of the form

$$
\begin{aligned}
T(z) & =\frac{b_{0} z^{n}+\cdots+b_{n-1} z+b_{n}}{z^{n}-a_{1} z^{n-1}-\cdots-a_{n-1} z-a_{n}} \\
& =D+\frac{\bar{b}_{1} z^{n-1}+\cdots+\bar{b}_{n-1} z+\bar{b}_{n}}{z^{n}-a_{1} z^{n-1}-\cdots-a_{n-1} z-a_{n}}
\end{aligned}
$$

with non-negative coefficients if Eqn. (21) has a nonnegative solution, i.e., $a \in \mathbb{R}_{+}^{n}$ and

$$
\bar{b}_{k}=\hat{g}_{k}-\sum_{i=1}^{k-1} \hat{g}_{k-i} a_{i} \geq 0, \quad k=1, \ldots, n .
$$

3.2. Solution of Subproblem 2. It is well known (Kaczorek, 2002; Benvenuti and Farina, 2004; Kaczorek, 2004; Kaczorek, 2006a; Kaczorek, 2006c) that if the coefficients $a_{1}, \ldots, a_{n}$ and $b_{0}, b_{1}, \ldots, b_{n}$ are nonnegative, then there always exists a positive realization (2) of the transfer function (25). For example, we may choose the positive realization as follows:

$$
\begin{gathered}
A=\left[\begin{array}{ccccc}
0 & 1 & 0 & \ldots & 0 \\
0 & 0 & 1 & \ldots & 0 \\
\ldots & \ldots & \ldots & \ldots & \ldots \\
0 & 0 & 0 & \ldots & 1 \\
a_{n} & a_{n-1} & a_{n-2} & \ldots & a_{1}
\end{array}\right], B=\left[\begin{array}{c}
0 \\
0 \\
\vdots \\
0 \\
1
\end{array}\right], \\
C=\left[\begin{array}{cccc}
\bar{b}_{n} & \bar{b}_{n-1} & \ldots & \bar{b}_{1}
\end{array}\right],
\end{gathered}
$$

or

$$
\begin{gathered}
\bar{A}=\left[\begin{array}{ccccc}
0 & 0 & \ldots & 0 & a_{n} \\
1 & 0 & \ldots & 0 & a_{n-1} \\
0 & 1 & \ldots & 0 & a_{n-2} \\
\ldots & \ldots & \ldots & \ldots & \ldots \\
0 & 0 & \ldots & 1 & a_{1}
\end{array}\right], \bar{B}=\left[\begin{array}{c}
\bar{b}_{n} \\
\bar{b}_{n-1} \\
\vdots \\
\bar{b}_{1}
\end{array}\right], \\
\bar{C}=\left[\begin{array}{llll}
0 & 0 & \ldots & 1
\end{array}\right], \\
\bar{D}=b_{0} .
\end{gathered}
$$

From (25) we have

$$
\begin{aligned}
D=b_{0}, \quad \bar{b}_{1} & =b_{1}+a_{1} b_{0}, \\
\bar{b}_{2} & =b_{2}+a_{2} b_{0}, \ldots, \bar{b}_{n}=b_{n}+a_{n} b_{0}
\end{aligned}
$$

and, using (24), we obtain (26).

Therefore, if the coefficients $a_{1}, \ldots, a_{n}$ and $b_{0}, b_{1}, \ldots, b_{n}$ are non-negative, then so are the coefficients $\bar{b}_{1}, \bar{b}_{2}, \ldots, \bar{b}_{n}$, and the realizations (27) are then positive. From (28) it follows that $\bar{b}_{k} \geq 0$ and $a_{k} \geq 0$ do not imply that $b_{k} \geq 0, k=1, \ldots, n$. 
If (21) has a non-negative solution $a \in \mathbb{R}_{+}^{n}$, then a positive realization (2) for a given finite sequence (19) can be computed using the following procedure:

\section{Procedure 1. (SISO systems)}

Step 1. Given a sequence (10), find a non-negative solution $a \in \mathbb{R}_{+}^{n}$ to (21).

Step 2. Using (24), compute the coefficients $b_{0}, b_{1}, \ldots, b_{n}$ and find the transfer function (25).

Step 3. Using one of the well-known methods (Benvenuti and Farina, 2004; Farina and Rinaldi, 2000; Kaczorek, 2002; Kaczorek, 2006a; Kaczorek, 2006b; Kaczorek, 2004; Kaczorek, 2005; Kaczorek, 2006c; Kaczorek, 2006d; Kaczorek, 2006e; Kaczorek and Busłowicz, 2004) compute a desired positive realization (2) of the transfer function (25), e.g., the positive realization (27).

Remark 1. If $n$ is not known a priori, then it is recommended to start the procedure with its small value and to increase it in the next step.

Example 1. Find a positive realization (2) for the sequence

$$
\hat{g}_{0}=1, \quad \hat{g}_{1}=3, \quad \hat{g}_{2}=8, \quad \hat{g}_{3}=19, \quad \hat{g}_{4}=46 .
$$

Using the procedure for $N=4$ and $n=2$, we obtain the following:

Step 1. In this case, Eqn. (21) for (29) has the form

$$
\left[\begin{array}{cc}
8 & 3 \\
19 & 8
\end{array}\right]\left[\begin{array}{l}
a_{1} \\
a_{2}
\end{array}\right]=\left[\begin{array}{l}
19 \\
46
\end{array}\right]
$$

and its solution is $a_{1}=2, a_{2}=1$.

Step 2. Using (24), we compute the coefficients

$$
\begin{aligned}
& b_{0}=\hat{g}_{0}=1, \quad b_{1}=\hat{g}_{1}-\hat{g}_{0} a_{1}=1, \\
& b_{2}=\hat{g}_{2}-\hat{g}_{1} a_{1}-\hat{g}_{0} a_{2}=1 .
\end{aligned}
$$

The desired transfer function has the form

$$
T(z)=\frac{z^{2}+z+1}{z^{2}-2 z-1}=1+\frac{3 z+2}{z^{2}-2 z-1}
$$

and

$$
\frac{3 z+2}{z^{2}-2 z-1}=3 z^{-1}+8 z^{-2}+19 z^{-3}+46 z^{-4}+\cdots
$$

Step 3. Using (27a) for (30), we obtain the desired positive realization

$$
\begin{aligned}
A=\left[\begin{array}{cc}
0 & 1 \\
a_{2} & a_{1}
\end{array}\right]=\left[\begin{array}{ll}
0 & 1 \\
1 & 2
\end{array}\right], & B=\left[\begin{array}{l}
0 \\
1
\end{array}\right], \\
C=\left[\begin{array}{ll}
\bar{b}_{2} & \bar{b}_{1}
\end{array}\right]=\left[\begin{array}{ll}
2 & 3
\end{array}\right], & D=[1] .
\end{aligned}
$$

Now let us assume that only the first four numbers of the sequence (29) are given, i.e., $N=3$ and $n=2$. In this case, Eqn. (21) takes the form

$$
8 a_{1}+3 a_{2}=19
$$

One of the coefficients $a_{1}, a_{2}$ can be chosen arbitrarily. If we choose $a_{1}=2$, then $a_{2}=1$, and we obtain the same transfer function (30) and its positive realization (31). If we choose $a_{1}=1$, then $a_{2}=11 / 3$ and $b_{0}=1, b_{1}=$ $2, b_{2}=4 / 3$.

The corresponding transfer function has the form

$$
T(z)=\frac{3 z^{2}+6 z+4}{3 z^{2}-3 z-11}=1+\frac{3 z+5}{z^{2}-z-\frac{11}{3}}
$$

and its positive realization is given by

$$
\begin{array}{cc}
A=\left[\begin{array}{cc}
0 & 1 \\
\frac{11}{3} & 1
\end{array}\right], & B=\left[\begin{array}{l}
0 \\
1
\end{array}\right], \\
C=\left[\begin{array}{ll}
5 & 3
\end{array}\right], & D=[1] .
\end{array}
$$

In this case

$$
\frac{3 z+5}{z^{2}-z-\frac{11}{3}}=3 z^{-1}+8 z^{-2}+19 z^{-3}+48 z^{-4}+\cdots
$$

Note that $g_{4}=48$ is different from $\hat{g}_{4}=46$ in the previous case.

Theorem 2. Let a finite sequence

$$
\hat{g}_{i}>0 \quad \text { for } \quad i=0,1, \ldots, 2 n
$$

be given. Then a positive realization of the form (27) exists if

$$
\begin{gathered}
\frac{\hat{g}_{n}}{\hat{g}_{n+1}}>\frac{\hat{g}_{n+1}}{\hat{g}_{n+2}}>\ldots>\frac{\hat{g}_{2(n-1)}}{\hat{g}_{2 n-1}}>\frac{\hat{g}_{2 n-1}}{\hat{g}_{2 n}}, \\
\frac{\hat{g}_{n}}{\hat{g}_{n+2}}>\frac{\hat{g}_{n+1}}{\hat{g}_{n+3}}>\ldots>\frac{\hat{g}_{2(n-1)}}{\hat{g}_{2 n}}>\frac{\hat{g}_{n-1}}{\hat{g}_{n+1}}, \\
\vdots \\
\frac{\hat{g}_{n}}{\hat{g}_{2 n}}>\frac{\hat{g}_{1}}{\hat{g}_{n+1}}>\ldots>\frac{\hat{g}_{n-2}}{\hat{g}_{2(n-1)}}>\frac{\hat{g}_{n-1}}{\hat{g}_{2 n-1}},
\end{gathered}
$$

and

$$
\bar{b}_{k}=\hat{g}_{k}-\sum_{i=1}^{k-1} \hat{g}_{k-i} a_{i} \geq 0, \quad k=1, \ldots, n,
$$

with $a_{i}, i=1, \ldots, n$, constituting the solution to (21) for $N=2 n$. 
Proof. Given a sequence (35), Eqn. (21) takes the form

$$
\begin{gathered}
{\left[\begin{array}{ccccc}
\hat{g}_{n} & \hat{g}_{n-1} & \ldots & \hat{g}_{2} & \hat{g}_{1} \\
\hat{g}_{n+1} & \hat{g}_{n} & \ldots & \hat{g}_{3} & \hat{g}_{2} \\
\ldots & \ldots & \ldots & \ldots & \ldots \\
\hat{g}_{2(n-1)} & \hat{g}_{2 n-3} & \ldots & \hat{g}_{n} & \hat{g}_{n-1} \\
\hat{g}_{2 n-1} & \hat{g}_{2(n-1)} & \ldots & \hat{g}_{n+1} & \hat{g}_{n}
\end{array}\right]\left[\begin{array}{c}
a_{1} \\
a_{2} \\
\vdots \\
a_{n-1} \\
a_{n}
\end{array}\right]} \\
=\left[\begin{array}{c}
\hat{g}_{n+1} \\
\hat{g}_{n+2} \\
\vdots \\
\hat{g}_{2 n-1} \\
\hat{g}_{2 n}
\end{array}\right] .
\end{gathered}
$$

Therefore,

$$
\left[\begin{array}{ccccc}
\frac{\hat{g}_{n}}{\hat{g}_{n+1}} & \frac{\hat{g}_{n-1}}{\hat{g}_{n+1}} & \ldots & \frac{\hat{g}_{2}}{\hat{g}_{n+1}} & \frac{\hat{g}_{1}}{\hat{g}_{n+1}} \\
\frac{\hat{g}_{n+1}}{\hat{g}_{n+2}} & \frac{\hat{g}_{n}}{\hat{g}_{n+2}} & \ldots & \frac{\hat{g}_{3}}{\hat{g}_{n+2}} & \frac{\hat{g}_{2}}{\hat{g}_{n+2}} \\
\ldots & \cdots & \ldots & \cdots & \cdots \\
\frac{\hat{g}_{2(n-1)}}{\hat{g}_{2 n-1}} & \frac{\hat{g}_{2 n-3}}{\hat{g}_{2 n-1}} & \cdots & \frac{\hat{g}_{n}}{\hat{g}_{2 n-1}} & \frac{\hat{g}_{n-1}}{\hat{g}_{2 n-1}} \\
\frac{\hat{g}_{2 n-1}}{\hat{g}_{2 n}} & \frac{\hat{g}_{2(n-1)}}{\hat{g}_{2 n}} & \ldots & \frac{\hat{g}_{n+1}}{\hat{g}_{2 n}} & \frac{\hat{g}_{n}}{\hat{g}_{2 n}} \\
\times\left[\begin{array}{c}
a_{1} \\
a_{2} \\
\vdots \\
a_{n-1} \\
a_{n}
\end{array}\right]=\left[\begin{array}{c}
1 \\
1 \\
\vdots \\
1 \\
1
\end{array}\right] . & & \\
\end{array}\right]
$$

If the conditions (36) are met, then the coefficient matrix of (38) has a cyclic structure and, by Theorem A (see the Appendix), Eqn. (38) has a positive solution $a_{i}>0$ for $i=1, \ldots, n$. In this case, using (37), we may find the coefficients $\bar{b}_{k}$ for $k=1, \ldots, n$ and the positive realization (27).

Example 2. (Continuation of Example 1)

For the sequence (29) we have that $N=2 n=4$ and (38) takes the form

$$
\left[\begin{array}{cc}
\frac{8}{19} & \frac{3}{19} \\
\frac{19}{46} & \frac{8}{46}
\end{array}\right]\left[\begin{array}{l}
a_{1} \\
a_{2}
\end{array}\right]=\left[\begin{array}{l}
1 \\
1
\end{array}\right] .
$$

It is easy to see that the coefficient matrix of (39) satisfies the conditions (36) since

$$
\frac{8}{19}>\frac{19}{46} \text { and } \frac{8}{46}>\frac{3}{19} \text {. }
$$

The transfer function has the form (30) and the desired positive realization is given by (31).

Theorem 3. A positive asymptotically stable realization (27) of the finite sequence (35) exists if

$$
\hat{g}_{i+1}<\hat{g}_{i} \quad \text { for } \quad i=1, \ldots, 2 n-1,
$$

and the conditions (36) and (37) satisfied.

Proof. If the conditions (36) are met, then by Theorem A Eqn. (38) has a positive solution $a_{i}>0$ for $i=1, \ldots, n$. We shall show that if (40) holds, then

$$
\sum_{i=1}^{n} a_{i}<1 .
$$

Let

$$
S_{i}=\sum_{j=1}^{n} \frac{\widehat{g}_{n-i+j}}{\widehat{g}_{n+j}}
$$

be the sum of the entries of the $i$-th column of the coefficient matrix in (38), $i=1, \ldots, n$. Adding the $n$ equations of (38), we obtain

$$
\sum_{i=1}^{n} S_{i} a_{i}=n .
$$

Note that if (40) holds, then each entry $\widehat{g}_{n-i+j} / \widehat{g}_{n+j}$ of the coefficient matrix is greater than 1. Write $\bar{S}=\min _{i} S_{i}$. Then from (42) we have

$$
\bar{S} \sum_{i=1}^{n} a_{i} \leq n
$$

and

$$
\sum_{i=1}^{n} a_{i} \leq \frac{n}{\bar{S}}<1
$$

since $\bar{S}>n$.

From the well-known final value theorem we obtain

$$
\begin{aligned}
g_{\infty} & =\lim _{i \rightarrow \infty} g_{i}=\lim _{z \rightarrow 1}(z-1) T(z) \\
& =\lim _{z \rightarrow 1}(z-1)\left[C\left[I_{n} z-A^{-1}\right] B+D\right] \\
& =\lim _{z \rightarrow 1}(z-1) \frac{b_{0} z^{n}+\ldots+b_{1} z+b_{0}}{z^{n}-a_{1} z^{n-1}-\cdots-a_{n-1} z-a_{0}}=0
\end{aligned}
$$

since (41) holds. Therefore, the positive realization (27) is asymptotically stable.

Example 3. We wish to find a positive realization (2) for the sequence

$$
\widehat{g}_{0}=0, \widehat{g}_{1}=1, \widehat{g}_{2}=0.9, \widehat{g}_{3}=0.6, \widehat{g}_{4}=0.5 \text {. }
$$


This sequence satisfies the conditions (40), (36) and (37) for $n=2$ and, by Theorem 3 , there exists a positive asymptotically stable realization. Using Procedure 1, we obtain the following:

Step 1. We have the system of equations

$\left[\begin{array}{cc}\frac{\widehat{g}_{2}}{\widehat{g}_{3}} & \frac{\widehat{g}_{1}}{\widehat{g}_{3}} \\ \frac{\widehat{g}_{3}}{\widehat{g}_{4}} & \frac{\widehat{g}_{2}}{\widehat{g}_{4}}\end{array}\right]\left[\begin{array}{l}a_{1} \\ a_{2}\end{array}\right]=\left[\begin{array}{cc}\frac{9}{6} & \frac{10}{6} \\ \frac{6}{5} & \frac{9}{5}\end{array}\right]\left[\begin{array}{l}a_{1} \\ a_{2}\end{array}\right]=\left[\begin{array}{l}1 \\ 1\end{array}\right]$

and its solution is $a_{1}=4 / 21, a_{2}=9 / 21$.

Step 2. Using (37), we compute the coefficients

$$
\bar{b}_{1}=\hat{g}_{1}=1, \bar{b}_{2}=\hat{g}_{2}-\hat{g}_{1} a_{1}=\frac{14.9}{21} .
$$

The desired transfer function has the form

$$
T(z)=\frac{z+\frac{14.9}{21}}{z^{2}-\frac{4}{21} z-\frac{9}{21}}=\frac{21 z+14.9}{21 z^{2}-4 z-9} .
$$

Step 3. Using (27a) for (44), we obtain the asymptotically stable realization

$$
\begin{aligned}
& A=\left[\begin{array}{cc}
0 & 1 \\
-a_{2} & -a_{1}
\end{array}\right]=\left[\begin{array}{cc}
0 & 1 \\
\frac{9}{21} & \frac{4}{21}
\end{array}\right], B=\left[\begin{array}{l}
0 \\
1
\end{array}\right], \\
& C=\left[\begin{array}{ll}
\bar{b}_{2} & \bar{b}_{1}
\end{array}\right]=\left[\begin{array}{ll}
\frac{14.9}{21} & 1
\end{array}\right], \quad D=[0] .
\end{aligned}
$$

\section{Extension for Multi-Input Multi-Output Systems}

The method presented in Section 3 can be easily extended for linear discrete-time systems with $m$ inputs and $p$ outputs (MIMO systems). In this case, the modified procedure has the following form:

Procedure 2. (MIMO systems)

Step 1. Given a sequence (6), find a non-negative solution

to (21) with

$$
a=\left[\begin{array}{llll}
a_{1} I_{m} & a_{2} I_{m} & \ldots & a_{n} I_{m}
\end{array}\right]^{T}
$$

$$
\begin{aligned}
\hat{G}_{n N} & =\left[\begin{array}{cccc}
\hat{G}_{n} & \hat{G}_{n-1} & \ldots & \hat{G}_{1} \\
\hat{G}_{n+1} & \hat{G}_{n} & \ldots & \hat{G}_{2} \\
\ldots & \ldots & \ldots & \ldots \\
\hat{G}_{N-1} & \hat{G}_{N-2} & \ldots & \hat{G}_{N-n}
\end{array}\right], \\
\hat{G}_{n N} & =\left[\begin{array}{c}
\hat{G}_{n+1} \\
\hat{G}_{n+2} \\
\vdots \\
\hat{G}_{N}
\end{array}\right] .
\end{aligned}
$$

Step 2. Using

$$
B_{k}=\hat{G}_{k}-\sum_{i=1}^{n} \hat{G}_{k-i} a_{i} \quad \text { for } \quad k=0,1, \ldots, n,
$$

compute the matrices $B_{0}, B_{1}, \ldots, B_{n}$ and the transfer matrix

$$
\begin{aligned}
T(z)= & \frac{1}{z^{n}-a_{1} z^{n-1}-\cdots-a_{n-1} z-a_{n}} \\
& \times\left[B_{0} z^{n}+B_{1} z^{n-1}+\ldots+B_{n-1} z+B_{n}\right] .
\end{aligned}
$$

Step 3. Using one of the well-known methods (Benvenuti and Farina, 2004; Farina and Rinaldi, 2000; Kaczorek, 2002; Kaczorek, 2006a; Kaczorek, 2006b; Kaczorek, 2004; Kaczorek, 2005; Kaczorek, 2006c; Kaczorek, 2006d; Kaczorek, 2006e; Kaczorek and Busłowicz, 2004) compute the desired positive realization (2) of the transfer matrix (47).

Example 4. We wish to find a positive realization (2) for the sequence

$$
\begin{array}{ll}
\hat{G}_{0}=\left[\begin{array}{l}
1 \\
2
\end{array}\right], & \hat{G}_{1}=\left[\begin{array}{l}
2 \\
3
\end{array}\right], \hat{G}_{2}=\left[\begin{array}{l}
5 \\
7
\end{array}\right], \\
\hat{G}_{3}=\left[\begin{array}{c}
7 \\
10
\end{array}\right], & \hat{G}_{4}=\left[\begin{array}{l}
12 \\
17
\end{array}\right] .
\end{array}
$$

We have $m=1$ and $p=2$. Using Procedure 2 for $N=4$ and $n=2$, we obtain the following:

Step 1. In this case, Eqn. (21) with (45) has the form

$$
\left[\begin{array}{cc}
5 & 2 \\
7 & 3 \\
7 & 5 \\
10 & 7
\end{array}\right]\left[\begin{array}{l}
a_{1} \\
a_{2}
\end{array}\right]=\left[\begin{array}{c}
7 \\
10 \\
12 \\
17
\end{array}\right]
$$

and its solution is $a_{1}=a_{2}=1$.

Step 2. Using (46), we compute the matrices

$$
\begin{aligned}
& B_{0}=\hat{G}_{0}=\left[\begin{array}{l}
1 \\
2
\end{array}\right], \hat{B}_{1}=\hat{G}_{1}-\hat{G}_{0} a_{1}=\left[\begin{array}{l}
1 \\
1
\end{array}\right], \\
& \hat{B}_{2}=\hat{G}_{2}-\hat{G}_{1} a_{1}-\hat{G}_{0} a_{2}=\left[\begin{array}{l}
2 \\
2
\end{array}\right] .
\end{aligned}
$$

The desired transfer matrix (47) has the form

$$
\begin{aligned}
T(z) & =\frac{1}{z^{2}-z-1}\left[\begin{array}{c}
z^{2}+z+2 \\
2 z^{2}+z+2
\end{array}\right] \\
& =\frac{1}{z^{2}-z-1}\left[\begin{array}{c}
2 z+3 \\
3 z+4
\end{array}\right]+\left[\begin{array}{l}
1 \\
2
\end{array}\right] .
\end{aligned}
$$


Step 3. Using (27a), we obtain the desired positive realization (2) of (50) in the form

$$
\begin{array}{ll}
A=\left[\begin{array}{ll}
0 & 1 \\
1 & 1
\end{array}\right], \quad B=\left[\begin{array}{l}
0 \\
1
\end{array}\right], \\
C=\left[\begin{array}{ll}
3 & 2 \\
4 & 3
\end{array}\right], \quad D=\left[\begin{array}{l}
1 \\
2
\end{array}\right] .
\end{array}
$$

\section{Concluding Remarks}

The partial realization problem for positive discrete-time linear systems has been formulated and solved. It was shown that it has a solution if Eqn. (21) has a non-negative solution and (26) holds (Theorem 1). A procedure for SISO and MIMO systems was proposed for the computation of positive partial realizations for a given finite sequence (10) (or (6)). The procedure was illustrated with four numerical examples. It was shown that if the conditions (40), (36) and (38) are satisfied, then there exists a positive asymptotically stable realization of the form (27).

The deliberations can be extended for linear continuous-time systems and for 2D linear systems (Kaczorek, 2002).

\section{Acknowledgment}

This work was supported by the Ministry of Science and Higher Education in Poland under Grant No. 3 T11A 006 27.

\section{References}

Benvenuti L. and Farina L. (2004): A tutorial on the positive realization problem. - IEEE Trans. Automat. Contr., Vol. 49, No. 5, pp. 651-664.

Farina L. and Rinaldi S. (2000): Positive Linear Systems: Theory and Applications. - Wiley, New York.

Ho B.L. and Kalman R.E. (1966): Effective construction of linear state-variable models from input/output functions. Regelungstechnik, Bd. 12, pp. 545-548.

Kaczorek T. (1992): Linear Control Systems, Vol. 1. — Taunton: Research Studies Press.

Kaczorek T. (2002): Positive $1 D$ and 2D Systems. — London: Springer.

Kaczorek T. (2004): Realization problem for positive discretetime systems with delay. - System Sci., Vol. 30, No. 4, pp. 17-30.

Kaczorek T. (2005): Positive minimal realizations for singular discrete-time systems with delays in state and control. Bull. Pol. Acad. Sci. Techn. Sci., Vol. 53, No. 3, pp. 293 298

Kaczorek T. (2006a): Realization problem for positive multivariable discrete-time linear systems with delays in the state vector and inputs. - Int. J. Appl. Math. Comput. Sci., Vol. 16, No. 2, pp. 169-174.
Kaczorek T. (2006b) A realization problem for positive continuous-time linear systems with reduced number of delay. - Int. J. Appl. Math. Comput. Sci., Vol. 16, No. 3, pp. 325-331.

Kaczorek T. (2006c): Computation of realizations of discretetime cone-systems. - Bull. Polish Acad. Sci. Techn. Sci., Vol. 54, No. 3, pp. 347-350.

Kaczorek T. (2006d): A realization problem for positive continuous-time systems with reduced numbers of delays.-Int. J. Appl. Math. Comput. Sci., Vol. 16, No. 3, pp. 101-107.

Kaczorek T. (2006e): Minimal positive realization for discretetime systems with state time-delays. - Int. J. Comput. Math. Electr. Eng. COMPEL, Vol. 25, No. 4, pp. 812-826.

Kaczorek T. and Busłowicz M. (2004): Minimal realization problem for positive multivariable linear systems with delay. - Int. J. Appl. Math. Comput. Sci., Vol. 14, No. 2, pp. 181-187.

Merzyakov J.I. (1963): On the existence of positive solutions of a system of linear equations. - Uspekhi Matematicheskikh Nauk, Vol. 18, No. 3, pp. 179-186 (in Russian).

\section{Appendix}

Definition A. A positive matrix

$$
\begin{aligned}
& A= {\left[\begin{array}{ccc}
a_{11} & \ldots & a_{1 n} \\
\ldots & \ldots & \ldots \\
a_{n 1} & \ldots & a_{n n}
\end{array}\right], a_{i j}>0, } \\
& i=1, \ldots, n ; j=1, \ldots, n,
\end{aligned}
$$

is called a matrix with a (strictly) cyclic structure if and only if

$$
a_{i i}>a_{i+1, i}>\cdots>a_{n i}>a_{1 i}>\cdots>a_{i-1, i}
$$

for $i=1, \ldots, n$.

For example, the matrix

$$
\left[\begin{array}{lll}
5 & 2 & 3 \\
3 & 8 & 1 \\
2 & 6 & 6
\end{array}\right]
$$

has a cyclic structure and the matrix

$$
\left[\begin{array}{lll}
5 & 2 & 3 \\
2 & 8 & 1 \\
3 & 6 & 6
\end{array}\right]
$$

does not have a cyclic structure since $a_{21}=2<a_{31}=3$.

Theorem A. (Merzyakov, 1963) The system of linear algebraic equations

$$
a_{i 1} x_{1}+a_{i 2} x_{2}+\cdots+a_{i n} x_{1}=1, \quad i=1, \ldots, n,
$$

with $a_{i j}>0, i, j=1, \ldots, n$ has a positive solution $x_{i}>$ $0, i=1, \ldots, n$ if and only if its coefficient matrix (Al) has a cyclic structure.

Received: 2 March 2007 
